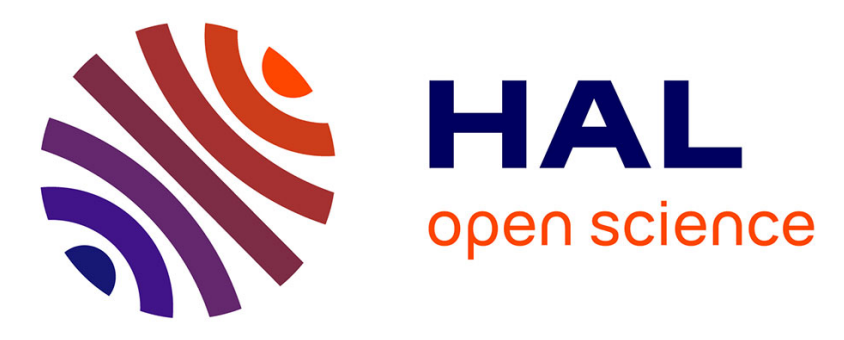

\title{
Determination of the optical constants of a dielectric layer by processing in situ spectral transmittance measurements along the time dimension
}

Michel Lequime, Severin Nadji, Cihan Koc, Dragan Stojcevski, Catherine Grezes-Besset, Julien Lumeau

\section{To cite this version:}

Michel Lequime, Severin Nadji, Cihan Koc, Dragan Stojcevski, Catherine Grezes-Besset, et al.. Determination of the optical constants of a dielectric layer by processing in situ spectral transmittance measurements along the time dimension. Applied optics, 2017, 56 (4), pp.C181-C187. 10.1364/AO.56.00C181 . hal-01424491

\section{HAL Id: hal-01424491 https://hal.science/hal-01424491}

Submitted on 13 Mar 2019

HAL is a multi-disciplinary open access archive for the deposit and dissemination of scientific research documents, whether they are published or not. The documents may come from teaching and research institutions in France or abroad, or from public or private research centers.
L'archive ouverte pluridisciplinaire HAL, est destinée au dépôt et à la diffusion de documents scientifiques de niveau recherche, publiés ou non, émanant des établissements d'enseignement et de recherche français ou étrangers, des laboratoires publics ou privés. 


\title{
Determination of the optical constants of a dielectric layer by processing in situ spectral transmittance measurements along the time dimension
}

\author{
Michel Lequime ${ }^{1, *}$, Séverin L. Nadji ${ }^{1}$, Dragan StojCevski ${ }^{1,2}$, Cihan Koc ${ }^{1}$, Catherine \\ GrÈZES-BESSET ${ }^{2}$, AND JULIEN LUMEAU ${ }^{1}$ \\ ${ }^{1}$ Aix Marseille Univ, CNRS, Centrale Marseille, Institut Fresnel, Marseille, France \\ ${ }^{2}$ CILAS, Etablissement de Marseille, Pole ALPHA Sud - Saint Mitre, 13400 Aubagne - France \\ ${ }^{*}$ Corresponding author : michel.lequime@fresnel.fr
}

Compiled November 14, 2016

This paper describes a new method based on the use of a broadband monitoring system to determine the spectral dependence of the optical constants of a layer without using a dispersion model. (๑) 2016 Optical Society of America

OCIS codes: (310.6860) Thin films, optical properties; (310.6188) Spectral properties.

http://dx.doi.org/10.1364/ao.XX.XXXXXX

\section{INTRODUCTION}

The knowledge of the spectral dependence of the optical constants [refractive index $n(\lambda)$ and extinction coefficient $\kappa(\lambda)$ ] of the materials involved in the manufacturing of an interference filter is a critical issue, especially during the design phase. During the manufacturing phase, one assumes that the deposition process is sufficiently repeatable to ensure that the optical constants of the deposited layers are the same as those used during the design, which is the case with energetic deposition processes, such as dual ion beam sputtering (DIBS) or plasma assisted reactive magnetron sputtering (PARMS).

Most of the time, to achieve this index determination [1], one begins by performing ex situ transmittance and reflectance measurements on a relatively thick (a few quarter wave optical thickness, QWOT) single layer deposited at the surface of a substrate characterized by a refractive index that is different from that of the layer under study [for instance, silica for high index materials such as tantala $\left.\left(\mathrm{Ta}_{2} \mathrm{O}_{5}\right)\right]$. Let us call $R_{\exp }$ and $T_{\text {exp }}$ the results of these measurements.

Thus one determines the theoretical data $R_{t h}$ and $T_{t h}$ by selecting a thin-film model (homogeneous or inhomogeneous) and a mathematical expression to describe the refractive index and extinction coefficient of the layer, such as the Cauchy and exponential laws for a slightly absorbing dielectric layer [2] :

$$
\left\{\begin{array}{l}
n(\lambda)=A_{0}+\frac{A_{1}}{\lambda^{2}}+\frac{A_{2}}{\lambda^{4}} \\
\kappa(\lambda)=B_{0} \exp \left(\frac{-B_{1}}{\lambda}\right) \exp \left(-B_{2} \lambda\right)
\end{array}\right.
$$

and, at the end, one minimizes a discrepancy function (DF) defined by [3] :

$$
\begin{aligned}
D F(\mathbf{X}, d)=\alpha \sqrt{\frac{1}{N} \sum_{n=1}^{N}\left[T_{t h}\left(\mathbf{X}, d, \lambda_{n}\right)-T_{\exp }\left(\lambda_{n}\right)\right]^{2}} \\
+\beta \sqrt{\frac{1}{N} \sum_{n=1}^{N}\left[R_{t h}\left(\mathbf{X}, d, \lambda_{n}\right)-R_{\exp }\left(\lambda_{n}\right)\right]^{2}}
\end{aligned}
$$

where $\alpha$ and $\beta$ are weighting factors in the range from 0 to $1, N$ is the number of wavelengths $\lambda_{n}$ for which spectral transmittance $T$ (or reflectance $R$ ) measurements are performed, $d$ is the layer thickness and $\mathbf{X}$ is a vector of dimension $m$ containing the $m$ parameters defining the index laws (here $m=6$ )..

The physical meaning of these dispersion laws can be improved by taking into account some fundamental constraints, such as causality, through the use of $n$ and $\kappa$ spectral dependence in accordance with Kramers-Kronig relations [4], but the final choice of a model includes a given arbitrariness. The objective of this paper is to show how the processing of the spectral transmittance data recorded by a broadband monitoring (BBM) system during the deposition of a single layer can be used to determine the spectral dependence of its optical constants without using a dispersion model.

Indeed, as illustrated in Fig. 1, a BBM system allows recording of the time evolution of the spectral transmittance $T(\lambda, t)$ of a substrate during the growth of a layer at its surface. These data can be processed along the wavelength dimension, for instance at the end of the deposition process, as previously described, but 
also along the time dimension at each wavelength $\lambda$ defined by the BBM system.

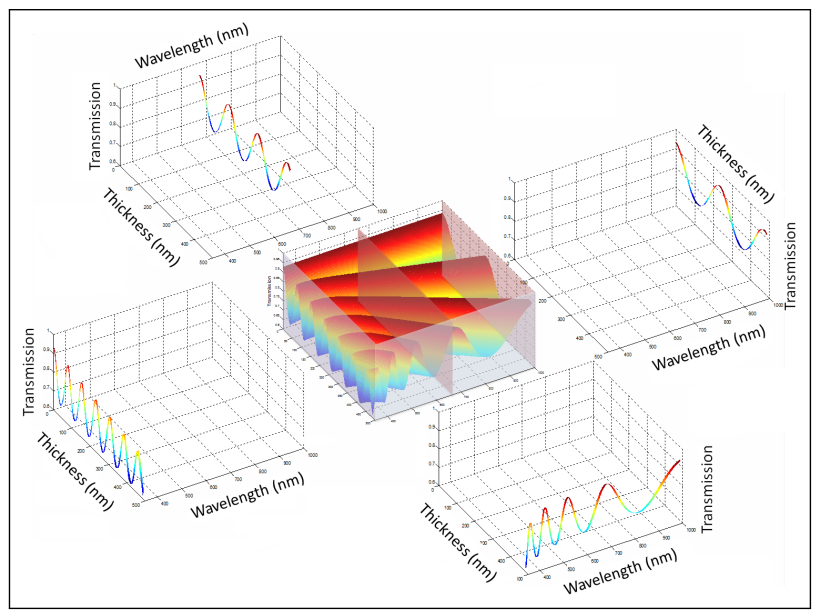

Fig. 1. BBM data (middle graph) processed along the wavelength (front graph) or time (rear and side graphs)dimensions.

If we assume that the rate of deposition $v$ of the layer is constant (this assumption is verified in the case of energetic deposition processes), the time period of the transmittance modulation at a given wavelength $\lambda$ is directly proportional to the refractive index $n(\lambda)$, while the time decrease of the transmittance envelope can be used to estimate the value of the extinction coefficient $\kappa(\lambda)$.

A brief description of the deposition machine and BBM system we used to demonstrate this concept is given in Section 2 of this paper, while Section 3 is devoted to a detailed description of our processing scheme. In Section 4, we analyze the quality of the data provided by this method and improve these results by applying refining steps. Then, we present and discuss the first results achieved in Section 5 and describe the content of further works.

\section{EXPERIMENTAL SET-UP}

The dual ion beam sputtering (DIBS) deposition machine selected for this experimental demonstration was manufactured in 2000 by Teer Coatings Ltd. (now a part of MIBA) and it implements two separate ion sources: the first providing a highly energetic argon ion beam that is aimed at a cooled metallic sputtering target (tantalum, hafnium or silicon) and the second, characterized by a lower energy ion beam, mixing argon and oxygen, is focused on the substrate and serves to correct the film stoichiometry and increase its packing density. Together these two sources operate to produce films with optimal optical and physical properties.

This deposition machine is equipped with a BBM system developed in our laboratory [5] whose schematic representation is shown in Fig. 2. The powerful and broadband light flux provided by a laser-driven light source (LDLS) from ENERGETIQ is launched into a $200 \mu \mathrm{m}$ diameter step index circular core fiber whose output extremity is located in the focal plane of an RC-04 reflective collimator from THORLABS. The collimated light beam crosses the sample installed in a rotating substrate holder (120 rpm) located at the top of the chamber (see Fig. 2). The transmitted light flux is sent into a $600 \mu \mathrm{m}$ step index square core fiber through an RC-12 reflective collimator, also from THORLABS.
A small part (10\%) of the coupled light is directed towards a linear photo-diode array spectrometer from TEC 5 (broadband monitoring or BBM channel) while the remaining part of the flux $(90 \%)$ passes through a Czerny-Turner spectrometer before detection by a single photodiode (monochromatic monitoring or MM channel).

At each turn of the substrate holder, the BBM system performs a fast acquisition ( $6 \mathrm{~ms}$ ) of three spectra over the wavelength range from $280 \mathrm{~nm}$ to $1020 \mathrm{~nm}$, the first corresponding to the light transmitted by the sample, the second to a reference measurement through a dedicated hole drilled in the substrate holder and the third to a dark measurement.

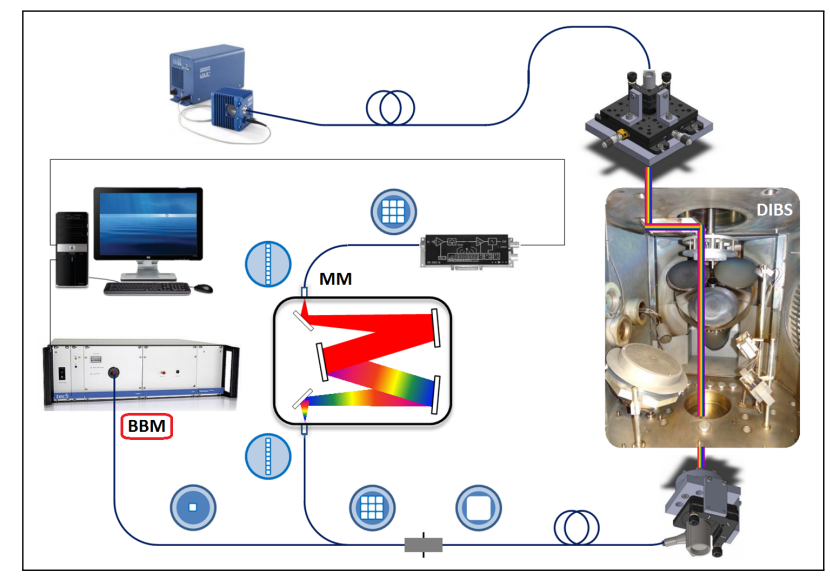

Fig. 2. Schematic representation of the DIBS chamber equipped with a dual optical monitoring system

In this way, we are able to record with good accuracy (typically better than $0.5 \%$ ) the time evolution of the spectral transmittance $T(\lambda, t)$ of the sample throughout the deposition process with a time interval of $0.5 \mathrm{~s}$ and a wavelength pitch of $0.8 \mathrm{~nm}$. Fig. 3 shows an example of the data recorded at $600 \mathrm{~nm}$ before (from 0 to approximately $1400 \mathrm{~s}$ ), during, and after (from $3400 \mathrm{~s}$ to $3500 \mathrm{~s}$ ) the deposition of a $7 \mathrm{H}$ tantala layer at the surface of a silica substrate.

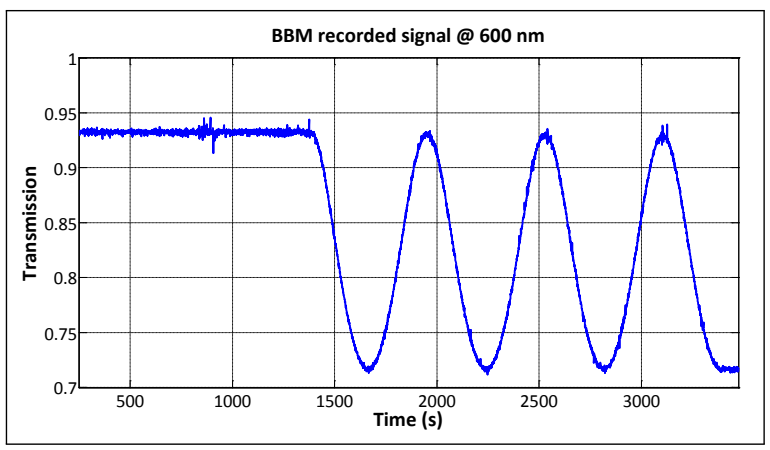

Fig. 3. Signal recorded by the BBM channel at $600 \mathrm{~nm}$ before, during, and after the deposition of a $7 \mathrm{H} \mathrm{Ta}_{2} \mathrm{O}_{5}$ layer.

\section{DESCRIPTION OF THE METHOD}

As defined above, we would like to determine the period of the transmittance time modulation for each wavelength recorded by the BBM channel. To achieve this goal with great accuracy, we begin by applying low-pass filtering to the signal recorded 
at wavelength $\lambda_{n}$ with a cut-off frequency $f_{c}$ equal to 8 times the modulation frequency of the transmittance at this specific wavelength.

Then, we perform derivation of the filtered signal and detect the time position $t_{k}$ of the zeros of this derivative, as shown in Fig. 4. Because of the frequency filtering, the derivative signal is very clean, and accordingly, the zeros detection is highly accurate.

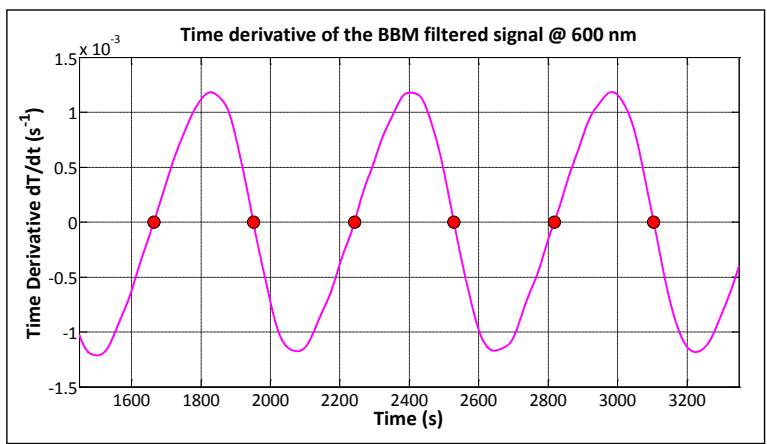

Fig. 4. Time derivative of the filtered signal at $600 \mathrm{~nm}$. (pink curve, time derivative of the transmittance; red dots, zeros of this derivative)

For each of these zeros, we have

$$
n_{1}\left(\lambda_{n}\right) v t_{k}=k \frac{\lambda_{n}}{4}
$$

where $n_{1}$ is the refractive index of the tantala layer, and $k$ is a positive integer. Accordingly, by plotting the QWOT's order $k$ with respect to time $t_{k}$, as shown in Fig. 5, we obtain a linear relationship whose slope $p\left(\lambda_{n}\right)$ is obtained through a least-square estimation and is defined by

$$
p\left(\lambda_{n}\right)=\frac{4 n_{1}\left(\lambda_{n}\right)}{\lambda_{n}} v
$$

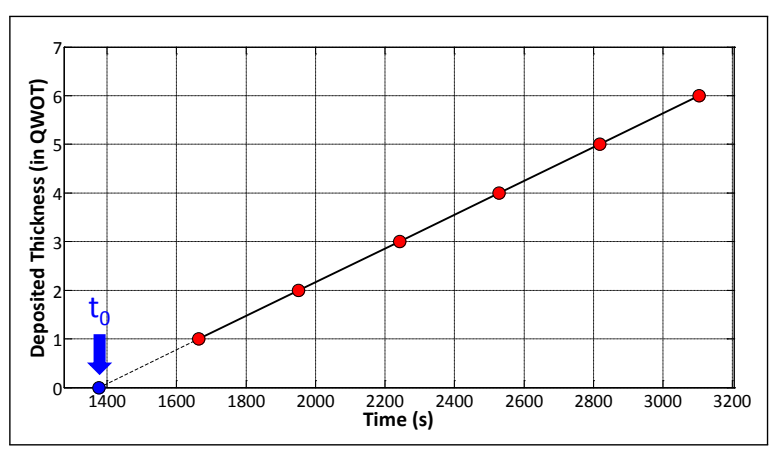

Fig. 5. Linear relationship between the QWOT's order and the time position of the zeros of the transmittance derivative. red dots, zeros of the transmission derivative; black straight line, linear fit; blue dot, starting time of the layer growth

The goodness of this linear fit is estimated by computing, for each wavelength $\lambda_{n}$, the coefficient of determination $R^{2}$ which is greater than 0.99987 in all cases. This result validates our prior assumption of the stability of the deposition rate $v$.

Moreover, through this linear fitting, one can estimate the start time of the layer growth $t_{0}$ (see Fig. 5). This determination can be achieved independently for each wavelength $\lambda_{n}$, which allows the computation of a mean value and a standard deviation for this start time $\left(t_{0}=1375.5 \pm 1.0 \mathrm{~s}\right)$.
The knowledge of all these slopes $p\left(\lambda_{n}\right)$ gives access to the refractive index dispersion $D\left(\lambda_{n}, \lambda_{0}\right)$ of the layer, i.e.

$$
D\left(\lambda_{n}, \lambda_{0}\right)=\frac{n_{1}\left(\lambda_{n}\right)}{n_{1}\left(\lambda_{0}\right)}=\frac{\lambda_{n}}{\lambda_{0}} \times \frac{p\left(\lambda_{n}\right)}{p\left(\lambda_{0}\right)}
$$

the use of a ratio allowing elimination of the unknown deposition rate $v$. Therefore, we need to know the value of the refractive index at a single wavelength $\lambda_{0}$ to complete the determination of $n_{1}(\lambda)$ at all the wavelengths $\lambda_{n}$. This can be achieved by computing, at this specific wavelength $\lambda_{0}$, the $T_{\min }\left(\lambda_{0}, t_{0}\right)$ transmission, which is defined (see Fig. 6) by the intercept between a linear fitting of the minima of transmission (green triangles in Fig. 6) and the vertical line corresponding to the mean starting time $t_{0}$

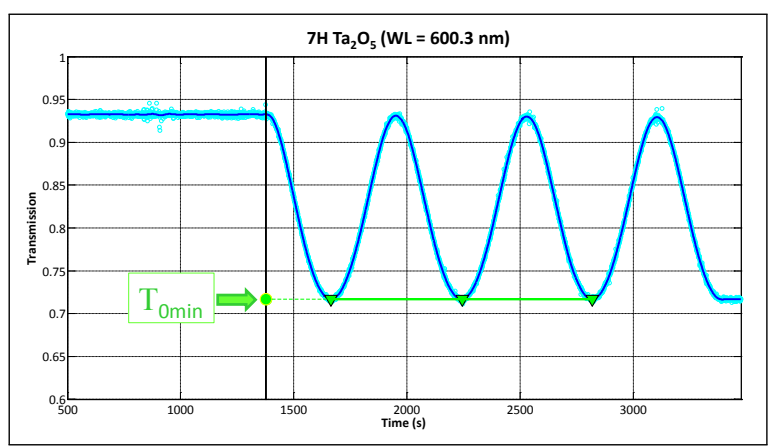

Fig. 6. Determination of the refractive index of the layer at $\lambda_{0}$ $=600.3 \mathrm{~nm}$

light blue curve, raw transmission data; dark blue curve, filtered transmission data; green triangles, minima of transmission; green straight line, linear fitting of the transmission minima; green dot, $T_{0}$ transmission

Basic computation shows that this $T_{\min }\left(\lambda_{0}, t_{0}\right)$ transmission is related to the unknown refractive index $n_{1}\left(\lambda_{0}\right)$ by the following relation:

$$
T_{\min }\left(\lambda_{0}, t_{0}\right)=\frac{4 n_{s}\left(\lambda_{0}\right) n_{1}^{2}\left(\lambda_{0}\right)}{\left[n_{s}^{2}\left(\lambda_{0}\right)+n_{1}^{2}\left(\lambda_{0}\right)\right]\left[1+n_{1}^{2}\left(\lambda_{0}\right)\right]}
$$

where $n_{S}$ is the refractive index of the silica substrate. For instance, at $600.3 \mathrm{~nm}: n_{\mathcal{S}}\left(\lambda_{0}\right)=1.4580, T_{\min }\left(\lambda_{0}, t_{0}\right)=0.7177$, and $n_{1}\left(\lambda_{0}\right)=2.1270$.

The refractive index at wavelength $\lambda_{0}$ provides access to the refractive index at all wavelengths $\lambda_{n}$ through simple multiplication by the refractive index dispersion $D\left(\lambda_{n}, \lambda_{0}\right)$, as shown in Fig. 7. This spectral dependence is quite smooth, except in

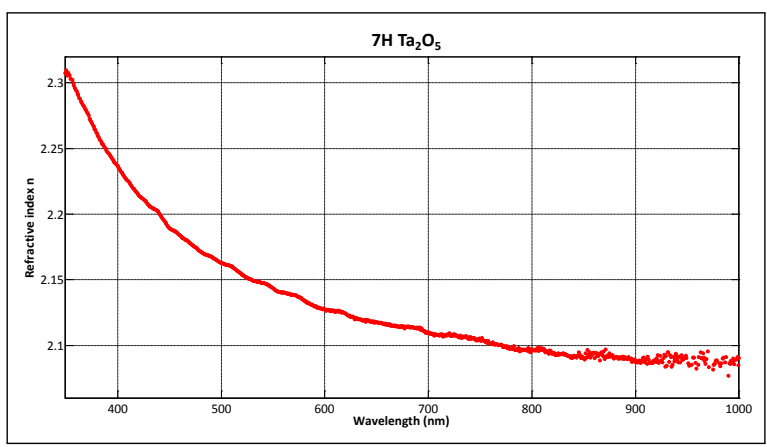

Fig. 7. Spectral dependence of the refractive index $n_{1}(\lambda)$ of the tantala layer. 
the infrared part of the spectrum, between $850 \mathrm{~nm}$ and $1000 \mathrm{~nm}$, where the data are more noisy.

The determination of the extinction coefficient $\kappa_{1}\left(\lambda_{n}\right)$ is achieved by computing the slope $s_{\max }\left(\lambda_{n}\right)$ of the linear decrease of the maxima of transmission with time, as shown in Fig. 8 in the blue part of the spectrum $\left(\lambda_{n}=419.7 \mathrm{~nm}\right)$.

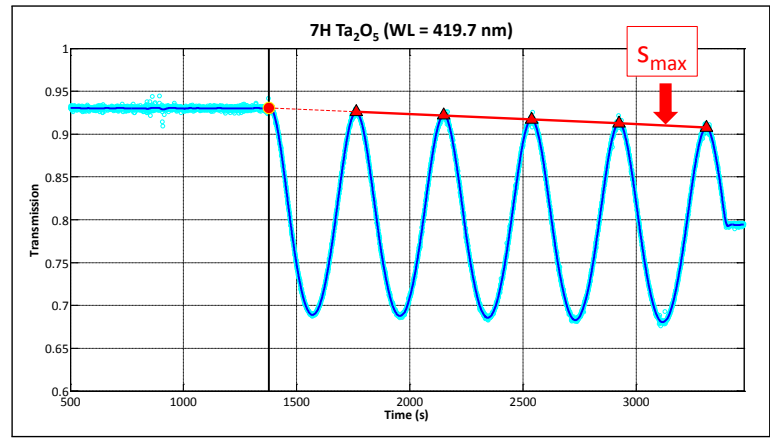

Fig. 8. Determination of the extinction coefficient of the layer at $\lambda_{n}=419.7 \mathrm{~nm}$.

light blue curve, raw transmission data; dark blue curve, filtered transmission data; red triangles, maxima of transmission; red straight line, linear fitting of the transmission maxima

Straightforward computation [5] shows that this slope is linked to the unknown extinction coefficient $\kappa_{1}\left(\lambda_{n}\right)$ by

$$
\begin{aligned}
& s_{\max }\left(\lambda_{n}\right)=-\frac{2 n_{s}\left(\lambda_{n}\right)}{1+n_{s}^{2}\left(\lambda_{n}\right)} \kappa_{1}\left(\lambda_{n}\right) \\
& \times \frac{\left[1+n_{s}\left(\lambda_{n}\right)\right]\left[n_{1}^{2}\left(\lambda_{n}\right)+n_{s}\left(\lambda_{n}\right)\right]}{n_{1}\left(\lambda_{n}\right)\left[1+n_{s}^{2}\left(\lambda_{n}\right)\right]} \frac{2 \pi}{\lambda_{n}} v
\end{aligned}
$$

Computing the ratio between $s_{\max }\left(\lambda_{n}\right)$ and $p\left(\lambda_{n}\right)$ allows, as previously, elimination of the deposition speed $v$, which leads to

$$
\begin{aligned}
\kappa_{1}\left(\lambda_{n}\right)=-\frac{1}{\pi} \frac{s_{\max }\left(\lambda_{n}\right)}{p\left(\lambda_{n}\right)} & \\
& \times \frac{n_{1}^{2}\left(\lambda_{n}\right)\left[1+n_{s}^{2}\left(\lambda_{n}\right)\right]^{2}}{n_{s}\left(\lambda_{n}\right)\left[1+n_{s}\left(\lambda_{n}\right)\right]\left[n_{1}^{2}\left(\lambda_{n}\right)+n_{s}\left(\lambda_{n}\right)\right]}
\end{aligned}
$$

The result is shown in Fig. 9 (blue circles).

As noted for the refractive index, the near infrared data are the noisiest because fewer extrema are used for the determination of the slopes $p\left(\lambda_{n}\right)$ and $s_{\max }\left(\lambda_{n}\right)$ in this part of the spectrum.

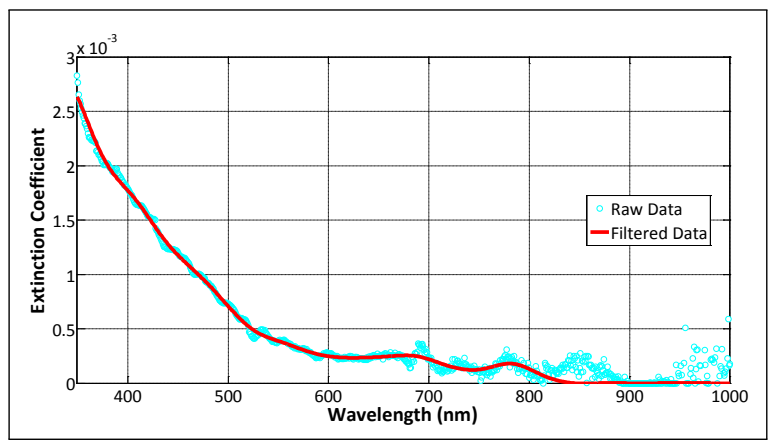

Fig. 9. Spectral dependence of the extinction coefficient $\bar{\kappa}_{1}(\lambda)$ of the tantala layer after filtering.

blue circles, raw data; red curve, filtered data

\section{DISCREPANCY FUNCTIONS AND REFINING}

We demonstrated that processing of the spectra recorded each $0.5 \mathrm{~s}$ along the time dimension using a BBM system during the deposition of a high index layer enables determination of the value of the optical constants of this layer by avoiding the choice of a dispersion model. This method also provides a highly accurate measurement of the deposition rate $(v=0.2447 \mathrm{~nm} / \mathrm{s})$, as well as a determination of the layer thickness at the end of the deposition $(d=494.7 \mathrm{~nm})$.

A spectral discrepancy function (SDF) can be used to estimate the quality of the determination provided by this new method. This SDF can easily be derived from relation (2) and is defined by

$$
\mathrm{SDF}=\sqrt{\frac{1}{N} \sum_{n=1}^{N}\left\{T_{t h}\left[d, n_{1}\left(\lambda_{n}\right), \kappa_{1}\left(\lambda_{n}\right)\right]-T_{\exp }\left(\lambda_{n}\right)\right\}^{2}}
$$

Fig. 10 shows the quality of the agreement between the spectral transmittance measurement at the end of the high-index layer deposition and the modeled data computed with the optical constants determined by our method.

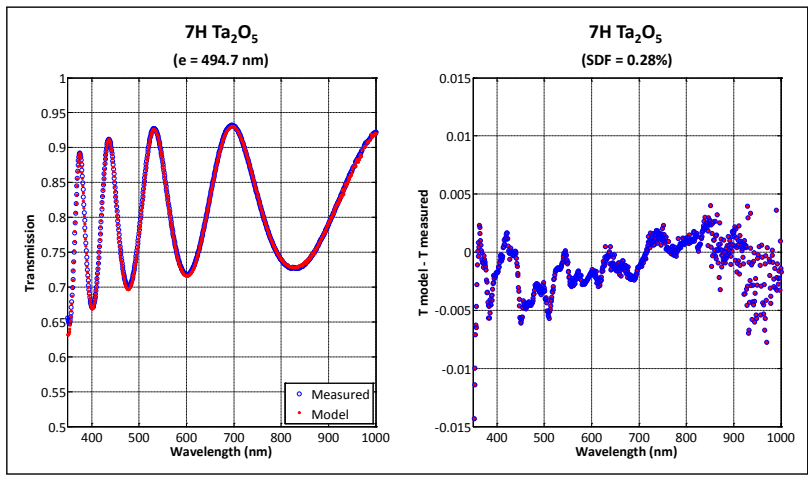

Fig. 10. Comparison of the experimental spectral transmittance measurements at the end of the high-index layer deposition and the modeled data computed with the optical constants determined by our method.

left graph: blue circles, measured data; red points, modeled data - right graph: residual discrepancy between measured and modeled data

The low value of the spectral discrepancy function $(\mathrm{SDF}=$ $0.28 \%$ ) is convincing proof of its efficiency. Two distinct ways were investigated to improve this result, i.e., first, filtering of the extinction coefficient data, and second, a refining step applied to the refractive index data along the time dimension.

\section{A. Extinction coefficient filtering}

As stressed in Section 3, the data obtained for the extinction coefficient are relatively noisy, especially in the near infrared part of the spectrum. Moreover, tantala layers deposited with an energetic process such as DIBS are perfectly transparent above $900 \mathrm{~nm}$. Therefore, it is physically meaningful to force the extinction coefficient to zero above this specific wavelength and to apply low-pass filtering to the corrected raw data with a cut-off frequency of $0.02 \mathrm{~nm}^{-1}$. The result of this processing step is shown in Fig. 9 (red curve).

If we replace, in the SDF expression (9), the raw data $\kappa_{1}\left(\lambda_{n}\right)$ with the filtered data $\bar{\kappa}_{1}\left(\lambda_{n}\right)$, we obtain exactly the same result $(0.28 \%)$. This is not surprising because the difference between the data are small, and the sensitivity of the layer transmittance 


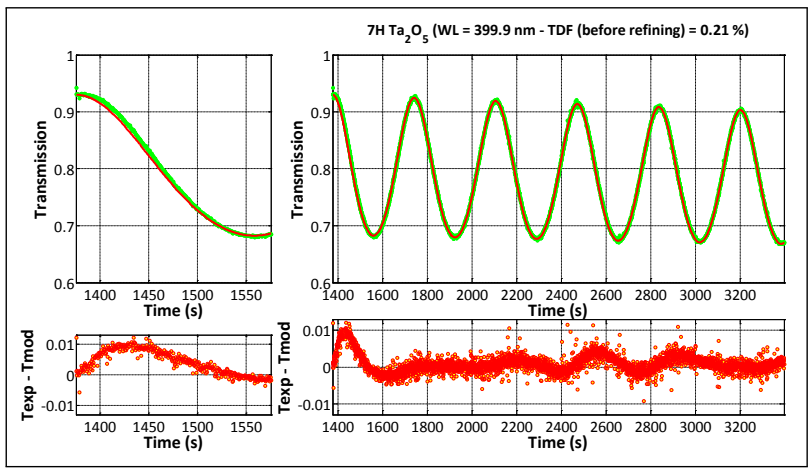

Fig. 11. Comparison between the time dependence of the transmittance measured at $\lambda_{n}=399.9 \mathrm{~nm}$ and the modeled data computed with the optical constants $n_{1}\left(\lambda_{n}\right)$ and $\bar{\kappa}_{1}\left(\lambda_{n}\right)$ determined by our method.

top graphs: green curve, experimental data - red curve, modeled data; bottom graphs: red circles, difference between experimental and modeled transmittance

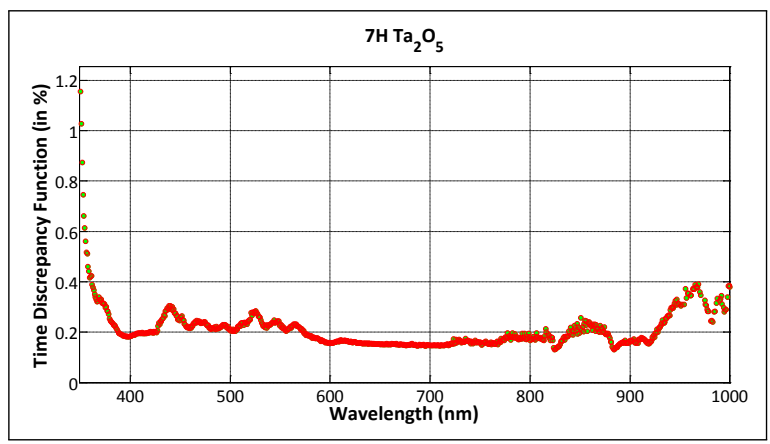

Fig. 12. Spectral dependence of the Time Discrepancy Function (in \%)

to extinction coefficient change is low when these changes are less than $10^{-4}$

\section{B. Refractive index refining}

For each wavelength $\lambda_{n}$, we can perform a comparison between the time dependence of the transmittance recorded by the BBM system $T_{\exp }\left(\lambda_{n}, t\right)$ and the modeled data $T_{t h}\left[v t, n_{1}\left(\lambda_{n}\right), \bar{\kappa}_{1}\left(\lambda_{n}\right)\right]$ computed using the last results of our determination. As previously, the quality of the agreement is quantified using a discrepancy function, defined here along the time dimension (TDF) by

$$
\operatorname{TDF}\left(\lambda_{n}\right)=\sqrt{\frac{1}{K} \sum_{k=1}^{K}\left\{T_{t h}\left[v t_{k}, n_{1}\left(\lambda_{n}\right), \bar{\kappa}_{1}\left(\lambda_{n}\right)\right]-T_{\text {exp }}\left(\lambda_{n}, t_{k}\right)\right\}^{2}}
$$

Fig. 11 shows that the quality of the agreement along the time dimension at $\lambda_{n}=399.9 \mathrm{~nm}$ (TDF $=0.21 \%$ ), is comparable to that obtained along the spectral dimension, at the end of the layer deposition (SDF $=0.28 \%$ ). The analysis of the TDF spectral dependence (see Fig. 12) shows that the agreement between the experimental and modeled data remains less than $0.3 \%$ in the main part of the spectrum.

To complete our processing sequence, we perform, for each wavelength $\lambda_{n}$, a final optimization of the time discrepancy function through a fine adjustment of the refractive index of the layer at this wavelength. Let us call $\bar{n}_{1}\left(\lambda_{n}\right)$ the new determination obtained after this refining step. The changes in refractive index

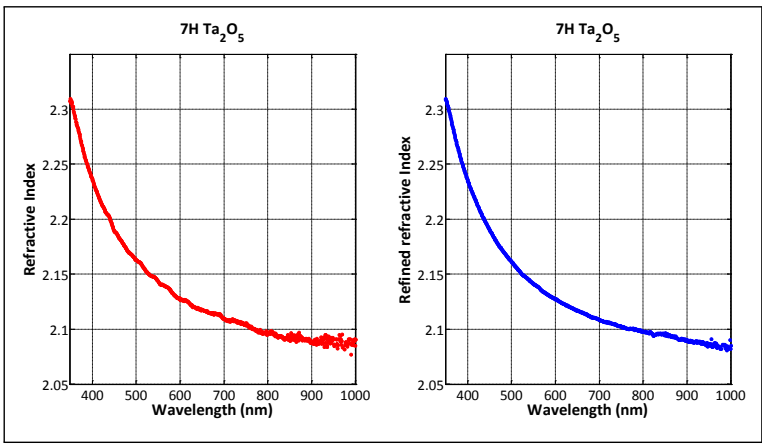

Fig. 13. Spectral dependence of the refractive index of the tantala layer.

left graph, red dots: before refining; right graph, blue dots: after refining

and TDF values it induces are small (respectively $0.0007 \pm 0.0021$ and less than $0.03 \%)$, as is the change in SDF $(0.24 \%$ instead of $0.28 \%$ ). The main benefit of this refining is a smoother spectral dependence of the refractive index, as illustrated in Fig. 13. It is important to consider that each value is obtained independently.

\section{DISCUSSION}

First of all, we can perform a comparison of the results provided by our method and by two standard cost function minimization methods, i.e.

- OptiChar, the special module of the OptiLayer software developed for the optical characterization of single thin films based on spectral photometric or/and ellipsometric measurements [7],

- the Global Optimization method recently proposed by Gao and Lemarchand [8].

The results are done in Table 1 and Fig. 14.

Table 1. Comparison of the results provided by 3 different methods

\begin{tabular}{lccccc}
\hline Method & $d(\mathrm{~nm})$ & $n_{1}(400)$ & $\kappa_{1}(400)$ & $n_{1}(1000)$ & SDF \\
\hline Optichar & 420.1 & 2.2556 & 0.0012 & 2.1008 & $0.55 \%$ \\
Gao & 421.2 & 2.2427 & 0.0010 & 2.0904 & $0.31 \%$ \\
This paper & 422.7 & 2.2325 & 0.0013 & 2.0871 & $0.37 \%$ \\
\hline
\end{tabular}

The three different determinations of the spectral dependence of the optical thickness are in excellent agreement, as expected. The slight difference between the determinations of the layer thickness $( \pm 0.3 \%)$ is indeed the only cause of the refractive index discrepancy. For the extinction coefficient, above $5.10^{-4}$, the agreement between the three methods is again very good. Below this threshold, the standard methods provide smoother wavelength dependence, but it is only a direct consequence of the use of a priori dispersion models. On the other hand, the SDF figure corresponding to our method is very close to the best one provided by the Gao-Lemarchand approach, that even so implements R and T measurements. Clearly, further investigations are required to conclude on the best way for achieving the determination of the very low extinction coefficient values (for 


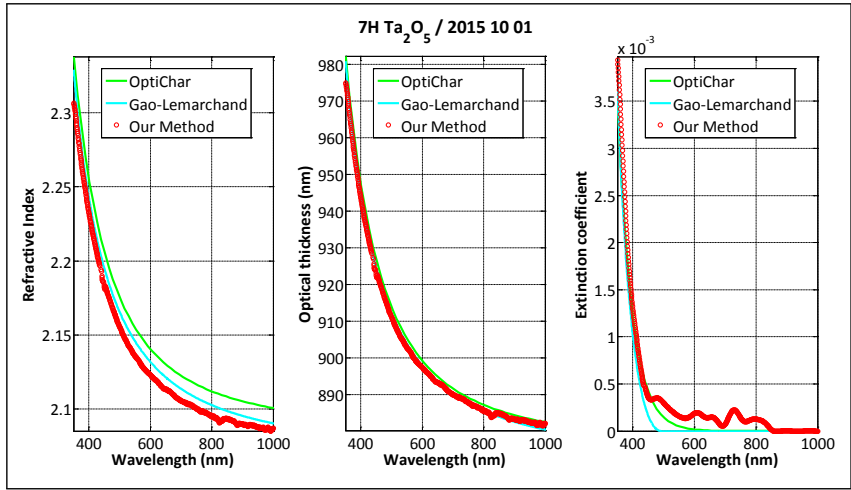

Fig. 14. Comparison of the results provided by three different methods (green curves, OptiChar software; cyan curves, GaoLemarchand method; red curves, our method)

at the left, spectral dependence of the refractive index; in the middle, spectral dependence of the optical thickness; at the right, spectral dependence of the extinction coefficient

instance, use of resonant layers, as the spacer of a Fabry-Perot cavity)

This provides a convincing proof that the processing of transmittance data recorded by a BBM system during the growth of a high-index layer along the time dimension is an effective way to determine the spectral dependence of its optical constants over a wide spectral range.

No a priori information about these spectral dependences, as embedded into Cauchy and/or exponential dispersion models, is required. Beside this main advantage, widely highlighted in this article, another noticeable one is the deterministic character of the data processing. Indeed, one can be confident that the spectral dependence of the optical constants it provides is, in any cases, close to the optimum solution. It is not always true with classical cost function minimization methods, for which human judgment and intervention are most of the time required, even in the case of Clustering Global Optimization [6]. It also means that the complete procedure (data recording and processing) can be implemented on line, through an entirely automatic way and just before the start of the manufacturing of a new filter.

Nevertheless, it obviously requires that the deposition machine is equipped with a highly accurate in situ BBM system and that the deposition technique is characterized by very stable deposition rates, as it is the case for energetic processes.

Moreover, our determination method provides an effective way to detect slight changes in the refractive index (or in the deposition rate) during the layer growth. Fig. 11 shows a positive difference of approximately 0.01 between the experimental transmittance and the modeled transmittance during the first 200 seconds of the deposition (corresponding thickness of approximately $50 \mathrm{~nm}$ ). This pattern, which is present at all wavelengths, is clearly not caused by acquisition noise or processing bias.

So, let us apply our TDF minimization procedure at a single wavelength (here $\lambda_{0}$ ), and for time intervals $\left[t_{0}, t\right]$ where $t$ increases regularly from $t_{0}+10$ seconds to the end of the layer deposition. For each time interval, and so, for each corresponding deposited thickness, we obtain a refined value of the refractive index at this specific wavelength. Figure 15 shows the evolution of this refractive index $\tilde{n}\left(\lambda_{0}\right)$ with the thickness of the layer, at the left over the first seventy nanometers and at the right during the whole growing of the layer.

One can see that the refractive index increases very rapidly from 1.651 for a thickness of $3 \mathrm{~nm}$ to 2.070 for a thickness of 20 $\mathrm{nm}$ before to reach a roughly constant value $(2.126 \pm 0.005)$ from $50 \mathrm{~nm}$. This evolution of the refractive index with the deposited

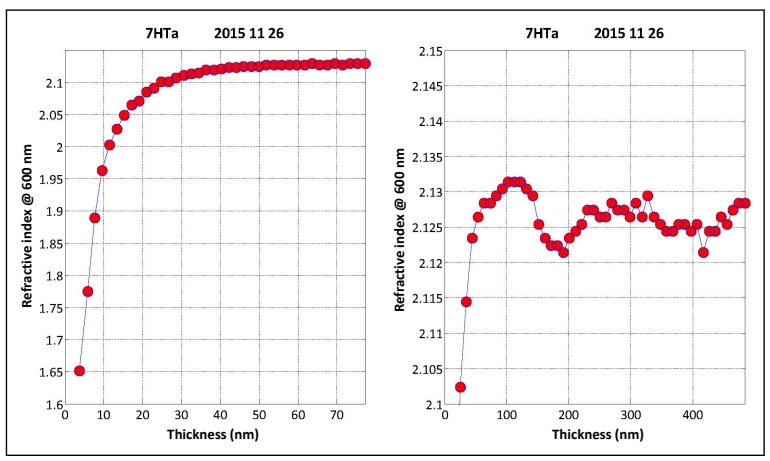

Fig. 15. Evolution of the refined refractive index $n_{1}\left(\lambda_{0}\right)$ with the thickness of the deposited layer

at the left, in the early time of the deposition; at the right, during the whole growing of the layer

thickness is in accordance with standard description of the layer growth mechanism [9].

However, at our level, it is very difficult to distinguish between refractive index evolution and deposition speed change, since the consequence on the thin-film optical properties are approximately the same. Further investigations, including mechanical thickness measurements and thin-film structure determination through white-light or atomic force microscopy, are thus required to definitively conclude on this point.

Finally, this method allows objective comparison of the repeatability of these optical constants for various deposition runs using the same process parameters as well as the influence of the deposition parameters on the refractive index and extinction coefficient of the layer.

Figures 16 and 17 highlight this ability for 5 deposition runs performed over 6 months (Process A: 4 runs; Process B: 1 run). The main characteristics of these 5 runs are summarized in Table 2. The refractive index dispersion remains less than 0.01 PTV

Table 2. Main characteristics of the deposition runs

\begin{tabular}{ccccc}
\hline Process & Date & $n_{1}(400)$ & $\kappa_{1}(400)$ & $n_{1}(1000)$ \\
\hline A & $2015 / 10 / 01$ & 2.2325 & 0.0013 & 2.0871 \\
A & $2015 / 10 / 02$ & 2.2424 & 0.0014 & 2.0926 \\
A & $2015 / 11 / 26$ & 2.2357 & 0.0018 & 2.0850 \\
A & $2016 / 02 / 24$ & 2.2381 & 0.0018 & 2.0865 \\
B & $2016 / 03 / 08$ & 2.2354 & 0.0029 & 2.0755 \\
\hline
\end{tabular}

for the same process, while the extinction coefficient is more strongly impacted by the process parameters change. 


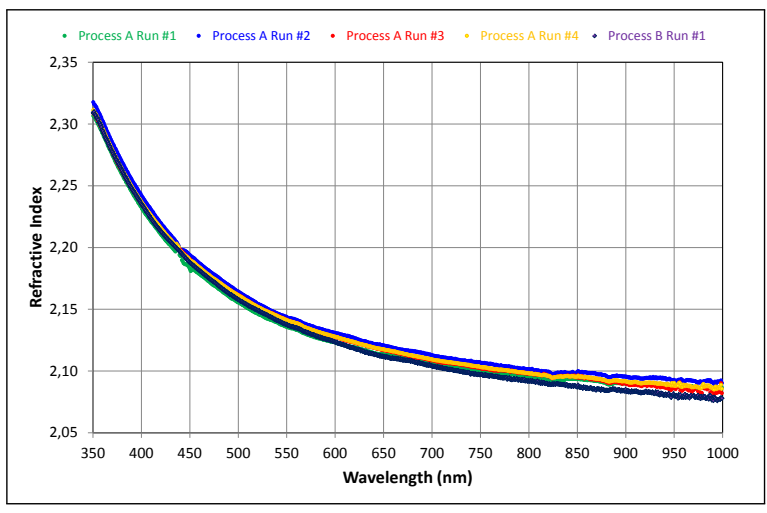

Fig. 16. Spectral dependence of the refractive index of a tantala layer for 5 deposition runs and 2 deposition processes. Process A, Run\#1, green dots; Process A, Run\#2, blue dots; Process A, Run\#3, red dots; Process A, Run\#4, yellow dots; Process B, Run\#1, violet diamonds

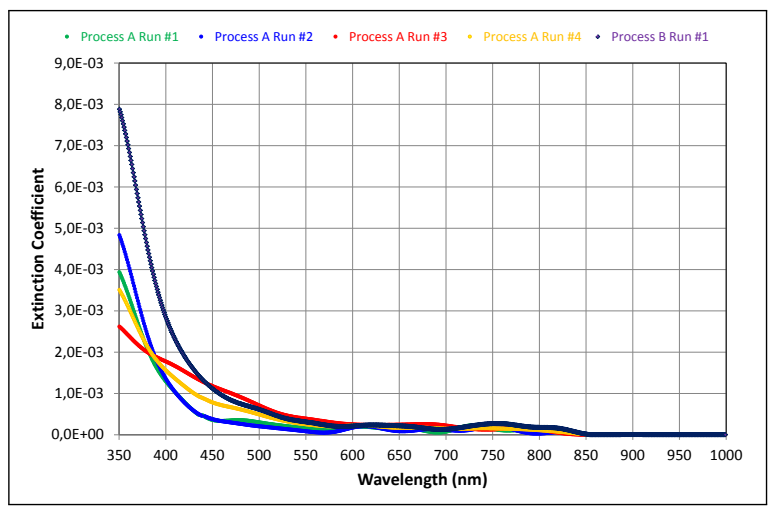

Fig. 17. Spectral dependence of the extinction coefficient of a tantala layer for 5 deposition runs and 2 deposition processes. Process A, Run\#1, green dots; Process A, Run\#2, blue dots; Process A, Run\#3, red dots; Process A, Run\#4, yellow dots; Process B, Run\#1, violet diamonds

Moreover, by replacing the silica substrate with a high-index glass window (for instance, HOYA LAH66, $n=1.7715 @ 600$ $\mathrm{nm}$ ), our method can be also used to determine the spectral dependence of the optical constants of a silica layer. However, a 2-layer configuration (silica substrate / H layer / B layer/ Air) can also be used to perform this determination. Theoretical analysis and experimental demonstration of these two possible schemes will be presented in a further publication [10].

\section{REFERENCES}

1. D. Poelman and P. F. Smet, "Methods for the determination of the optical constants of thin films from single transmission measurements: a critical review," J. Phys. D: Appl. Phys. 36, 1850-1857 (2003)

2. A. V. Tikhonravov, M. K. Trubetskov, T. V. Amotchkina, G. DeBell, V. Pervak, A. K. Sytchkova, M. L. Grilli, D. Ristau, "Optical parameters of oxide films typically used in optical coating production," Appl. Opt. 50, C75-C85 (2011).

3. J. A. Dobrowolski, F. C. Ho, and A. Waldorf, "Determination of optical constants of thin film coating materials based on inverse synthesis," Appl. Opt. 22, 3191-3200 (1983).

4. L. Gao, F. Lemarchand, and M. Lequime, "Comparison of different dispersion models for single layer optical thin film index determination," Thin Solid Films 520, 501-509 (2011).

5. D. Stojcevski, "Développement d'un contrôle optique multicritère - Application à la détermination d'indice in situ, $\mathrm{PhD}$ Thesis, Aix-Marseille Université (2015).
6. F. Lemarchand, "Application of clustering global optimization to thin film design problems," Opt. Express 22, 5166-5176 (2014)

7. http://www.optilayer.com/products-and-services/optichar

8. L. Gao, F. Lemarchand, and M. Lequime, "Refractive index determination of $\mathrm{SiO}_{2}$ layer in the UV/Vis/NIR range: spectrophotometric reverse engineering on single and bi-layer designs," JEOS-RP 8, 13010 (2013).

9. N. Kaiser, "Review of the fundamentals of thin-film growth," Appl. Opt. 41, 3053-3060 (2002)

10. S. L. Nadji, M. Lequime, T. Begou, C. Koc, C. Grezes-Besset, and J. Lumeau are preparing a manuscript to be called "Use of a broadband monitoring system for the determination of the optical constants of a dielectric bilayer" 